\title{
BioOne

\section{A Phylogenetic Analysis of the Evolution of Austronesian Sibling Terminologies}

\author{
Author(s): Fiona M. Jordan \\ Source: Human Biology, 83(2):297-321. 2011. \\ Published By: Wayne State University Press \\ DOI: $10.3378 / 027.083 .0209$ \\ URL: http://www.bioone.org/doi/full/10.3378/027.083.0209
}

BioOne (www.bioone.org) is an electronic aggregator of bioscience research content, and the online home to over 160 journals and books published by not-for-profit societies, associations, museums, institutions, and presses.

Your use of this PDF, the BioOne Web site, and all posted and associated content indicates your acceptance of BioOne's Terms of Use, available at www.bioone.org/page/terms_of_use.

Usage of BioOne content is strictly limited to personal, educational, and non-commercial use. Commercial inquiries or rights and permissions requests should be directed to the individual publisher as copyright holder. 


\title{
A Phylogenetic Analysis of the Evolution of Austronesian Sibling Terminologies
}

FIONA M. JORDAN ${ }^{1}$

\begin{abstract}
Social structure in human societies is underpinned by the variable expression of ideas about relatedness between different types of kin. We express these ideas through language in our kin terminology: to delineate who is kin and who is not, and to attach meanings to the types of kin labels associated with different individuals. Cross-culturally, there is a regular and restricted range of patterned variation in kin terminologies, and to date, our understanding of this diversity has been hampered by inadequate techniques for dealing with the hierarchical relatedness of languages (Galton's Problem). Here I use maximum-likelihood and Bayesian phylogenetic comparative methods to begin to tease apart the processes underlying the evolution of kin terminologies in the Austronesian language family, focusing on terms for siblings. I infer (1) the probable ancestral states and (2) evolutionary models of change for the semantic distinctions of relative age (older/younger sibling) and relative sex (same-sex/opposite-sex). Analyses show that early Austronesian languages contained the relative-age, but not the relative-sex distinction; the latter was reconstructed firmly only for the ancestor of Eastern Malayo-Polynesian languages. Both distinctions were best characterized by evolutionary models where the gains and losses of the semantic distinctions were equally likely. A multi-state model of change examined how the relative-sex distinction could be elaborated and found that some transitions in kin terms were not possible: jumps from absence to heavily elaborated were very unlikely, as was piece-wise dismantling of elaborate distinctions. Cultural ideas about what types of kin distinctions are important can be embedded in the semantics of language; using a phylogenetic evolutionary framework we can understand how those distinctions in meaning change through time.
\end{abstract}

Social structure in human societies is underpinned by the variable expression of ideas about relatedness between kin. Notions of marriageability, determination of group membership, rules of residence and reciprocal obligations, and theories of child-rearing influence much of how human communities have structured their interactions. Anthropologists have long noted that while on the surface there is

${ }^{1}$ Evolutionary Processes in Language and Culture, Max Planck Institute for Psycholinguistics, PB 310, Nijmegen $6500 \mathrm{AH}$, The Netherlands.

Human Biology, April 2011, v. 83, no. 2, pp. 297-321.

Copyright (C) 2011 Wayne State University Press, Detroit, Michigan 48201-1309

KEY WORDS: KIN TERMS, LANGUAGE, AUSTRONESIAN, PHYLOGENETIC COMPARATIVE METHOD, CULTURAL EVOLUTION. 
a great deal of cross-cultural diversity in kinship systems, this diversity does not span the entirety of the variety that could exist (Nerlove and Romney 1967). This makes evolutionary sense, as kinship systems provide the environment in which humans mate, produce, and rear offspring, thus necessitating strong selective pressures against systems that might create non-adaptive environments. Humans are also cooperative breeders (Hrdy 2009), and so individuals within a group must coordinate their expectations and actions with respect to kin.

One of the most important ways we coordinate these expectations is by using language: first to delineate who is kin and who is not, and then second to attach meanings to the types of kin labels associated with different individuals (e.g., "mother") or classes of individuals (e.g., "cousin"). Within a language, the set of terms that comprise these kin relationships is called a kinship terminology. These terms vary between languages, as do lexical items generally, according to ancestor-descendant relationships; the more closely related two languages are, the more likely they are to share cognate forms. Thus in the Indo-European language family, the English word "sister," Dutch "zuster," German "Schwester," and Italian "sorella" are all derived from a common source and all denote a female sibling.

Closely related languages are also likely to share similar meanings for their terms, and the closer the relationship, the more likely it may be that meanings coincide. However, and like some other classes of words, kin terms have the property that meaning can be extended. That is, while in a language the word for "mother" may have some primary referent of the female parent, it may also extend to cover other female relatives of the ascending generation such as mother's sister. These patterns of extension vary cross-linguistically-some languages may have ten different terms to describe a set of kin, while others may have 40 (Fox 1994) - but there is a regular and restricted range of patterned variation. This paper will address the broad question of how these cultural meanings and linguistic forms evolve in the domain of kinship.

Comparative Studies of Kinship. Historically, the study of kin terminologies is foundational in anthropology (Morgan 1871), as is kinship more generally. The cross-cultural study of kinship was in effect abandoned or delegitimized by the last generation of anthropological scholars concerned with postmodernist qualms regarding relativity, comparison, and "biologizing" (Colleran and Mace 2011; D'Andrade 2000). This left the study of kin terminologies largely to linguists and cognitive anthropologists (e.g., Lounsbury 1987; Wierzbicka 1987), often scholars promoting increasingly algebraic approaches (e.g., Kronenfeld 2001; Read 2001, 2007) who have made the field forbidding to the non-specialist. During this period, the emergence of modern evolutionary anthropology (see e.g., Dunbar and Barrett 2007; Laland and Brown 2002) has put Darwinian kinship center-stage through the investigation of topics such as parental investment and inclusive fitness maximization, but these 
studies have rightly had the individual as the locus of investigation and are often not explicitly cross-cultural in design.

One strand of modern evolutionary anthropology has turned a lens back on comparative studies of kinship: cultural phylogenetics. By using the phylogenetic methods used by evolutionary biologists interested in cross-species diversity (Harvey and Pagel 1991), this approach seeks to understand evolutionary processes that produce patterned variation in culture (Gray et al. 2007; Mace and Holden 2005). In anthropology, the historical nonindependence of related populations has been recognized as a crucial difficulty for comparative analysis since Galton (Tylor 1889), but the statistical techniques used for comparative analysis of hierarchically related taxa in biology have only been adopted by cultural researchers in the last 15 years (Lipo et al. 2006; Mace et al. 2005; Mace and Pagel 1994). Recent studies have used phylogenetic tree- and networkbuilding methods on language data to investigate the population histories of major language families such as Austronesian (Gray et al. 2009), Bantu (Holden 2002; Rexova et al. 2006), Indo-European (Gray and Atkinson 2003), Chinese (Ben Hamed 2005) and Semitic (Kitchen et al. 2009). These methods have also been used to study cultural transmission in aspects of material culture (e.g., Jordan and Shennan 2009; Tehrani and Collard 2009).

Once constructed, these phylogenies can then be used as principled controls for the evolutionary history of populations, a scaffolding on which to test cross-cultural hypotheses. Comparative techniques can test hypotheses about correlated change (e.g., the coevolution of pastoralism and patrilineality in the Bantu [Holden and Mace 2003], or marriage forms and payments in Indo-European [Fortunato and Mace, 2009]). The methods can reconstruct ancestral states of culture (e.g., matrilocal residence in ancestral Austronesian societies [Jordan et al. 2009]), they can infer relative rates of cultural change (e.g., in different parts of the lexicon [Pagel et al. 2007]), and can be used to investigate patterns of descent and exchange-as seen in work on material culture traditions in Iranian textiles (Collard et al. 2006; Tehrani and Collard 2009).

Notably, kinship has been the focus of much phylogenetic comparative work. Kinship sits at an unparalleled nexus of biology, culture, and language. Concerned on one level with the regulation of human reproduction-the "basic facts of life" (Fox 1967) - kinship is more than biology, as crossculturally the structure, rights, and obligations of these social relationships show variable mapping onto genealogy; similarly, diversity in kinship structure is mediated through and expressed in language. How these elements come together to create the observed patterns of human cultural variation is an open question.

Here I use comparative phylogenetic methods to begin to tease apart the processes underlying the evolution of kin terminologies. The paper presents an analysis of the types of distinctions made in Austronesian terms for siblings, by reconstructing the probable ancestral states of distinctions based on relative age, 
Table 1. Examples of Austronesian Sibling Terminologies and the Distinctions Made by Meanings

\begin{tabular}{llll} 
Language & Term & Meaning & Distinctions \\
\hline Toba Batak & haha & Older same-sex sibling & Relative age, relative sex \\
& angi & Younger same-sex sibling & Relative age, relative sex \\
& i-boto & Opposite-sex sibling & Relative sex \\
Malagasy & ralahavy & Man's brother & Relative sex, sex of speaker \\
& rahavavy & Woman's sister & Relative sex, sex of speaker \\
& anadahy & Man's sister & Relative sex, sex of speaker \\
& anabavy & Woman's brother & Relative sex, sex of speaker \\
Ponape & riei & Sibling & None \\
\hline
\end{tabular}

Source: Blust (1994).

relative sex, and sex of speaker. Siblings here are formally defined as offspring, or persons recognized as such, of the same parent(s). Sibling terminologies, concerned as they are with the immediate relatives in ego's own generation, are a useful partial set of all possible kin with which to strategically investigate evolutionary processes, because they can vary within otherwise equivalent systems whose structure (outside of the sibling set) may reflect aspects of marriageabillity or lineal group membership (cf Levi-Strauss 1969; RadcliffeBrown 1952). Table 1 contains examples of sibling terms in Austronesian languages that demonstrate these distinctions. It should be noted that the familiar "sex of referent only" form in English (i.e., two terms comprising "brother," "sister" only) is barely attested in Austronesian.

These distinctions have been widely considered salient in the kinship terminology literature since Kroeber (1909; see also Parkin 1997), and are part of a constrained set of considerations including group membership (e.g., male/female, in-/out-group), genealogical distance (e.g., older/younger, levels of generation), and social rank that Jones (2003, 2010) has described as candidates for a species-typical, flexibly generative psychology of kinship. Though these ideas are largely untested, theoretically they derive from evolutionarily relevant considerations such as kin selection, the likelihood of altruistic behavior, and aspects of our primate heritage: they can thus be considered a highly appropriate starting point from a Darwinian perspective. However, these start points do not wed us to any particular biological or genealogical perspective on kinship; kinship is everywhere an "ideology of human relationships" (Stone 2000) where simple genetic relatedness is only part of the picture.

The Austronesian Context. The sibling terminologies analyzed here are drawn from data on the Austronesian (AN) languages of the Pacific, collected together by Dziebel (2009). This language family is spread across the Pacific from Taiwan to Polynesia, excluding Australia and most of New Guinea and surrounding islands (see Figure 1). Archaeological and linguistic work has reached a broad consensus that Austronesian dispersal began around $5500 \mathrm{BP}$ from Taiwan, with a corresponding ancestral speech community, Proto 
a

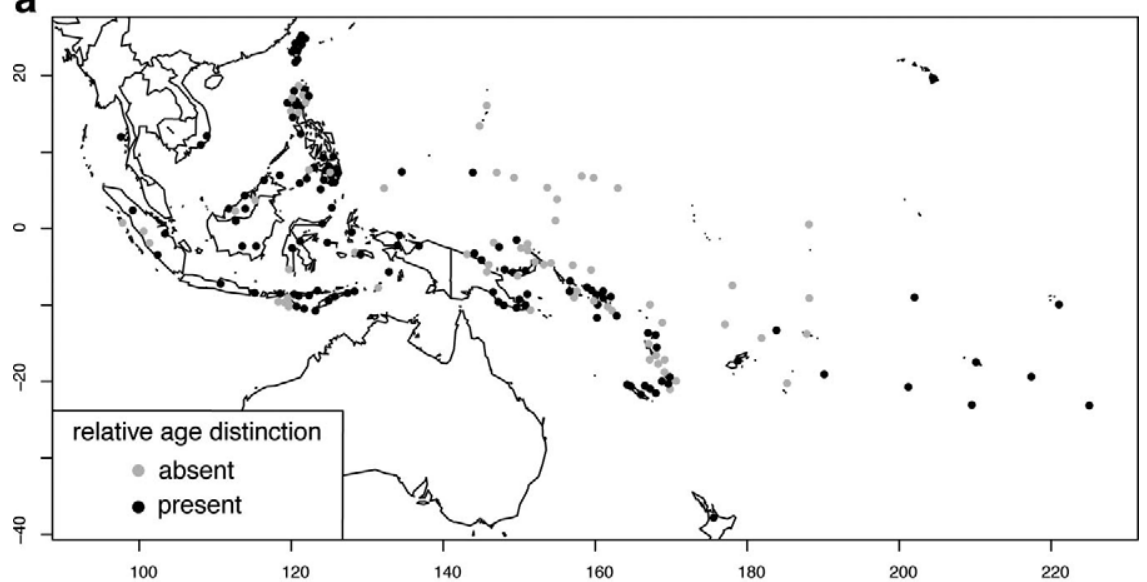

b

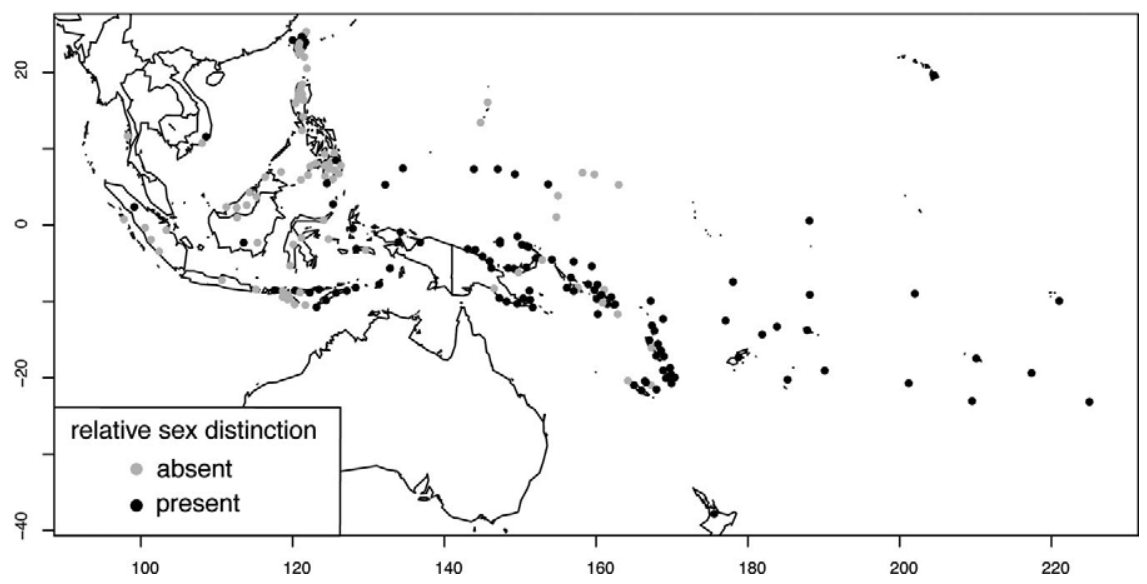

Figure 1. Map of the Pacific showing the location of the 208 languages used in the analyses. (a) The distribution of languages with a relative age distinction (present $=$ black circles, absent $=$ grey circles). (b) The distribution of languages with a relative sex distinction (present $=$ black circles, absent $=$ grey circles).

Austronesian (PAN) (Diamond and Bellwood 2003); entry into the Philippines and island Southeast Asia began around c. 4500 вP (Pawley 2002). This branching coincides with Proto Malayo-Polynesian (PMP), and then another later branching of Proto Oceanic (POC) around 3500 BP, associated with the archaeological "Lapita Cultural Complex" and the dispersal of Austronesian peoples into the previously uninhabited regions of Remote Oceania such as Polynesia (Green 2003; Kirch and Green 2001). Austronesian societies vary in ways representative of worldwide diversity in kinship (Fox 1994; Lane 1961). Murdock (1968) found Malayo-Polynesian languages to be of particular note in their complexity: his "Type F: Melanesian" class was the only type 
to be further divided into four subtypes. This diversity, combined with well-established models of population history, provides a useful regional case to test hypotheses.

Linguists and anthropologists have debated the nature of Austronesian kin terminologies for many years, often focusing on sibling terms (Blust 1980, 1994; Epling et al. 1973; Firth 1936; Fox et al. 1995; Marshall 1983, 1984; Murdock 1968; Van Wouden 1935, 1968). Here I briefly describe two points from this literature that inform the analyses below: the concept of an evolutionary model of change, and linguistic inferences about ancestral states. Epling et al. (1973) and Marshall (1984), drawing on Murdock (1968) and Nerlove and Romney (1967), used typological schemes to classify patterns of sibling terms. Thus, languages that had a single term "sibling" were one type, while languages with two terms denoting "older sibling" and "younger sibling" constituted another type, regardless of historical relationship. Both Epling et al. and Marshall proposed sequential models of change in these structural types as a way of explaining the diversity of sibling classifications in Polynesian and Oceanic respectively. Both authors also suggested that appropriate models of change were those in which binary distinctions (e.g., male/female) were added or removed to a type (e.g., older sibling/younger sibling) to create the next pattern in the sequence (e.g., older brother/older sister/younger sibling). However, these sequential models weighted all changes as equally probable and thus enforced stepwise increments in the distinctions- "jumps" were not possible. Linguists will be familiar with this "types and distinctions" contrast as similar to "paradigms and features"; that is, whether the locus of evolutionary investigation is more correctly at a system or a component level. This is an unanswered question with kinship: to date, no investigation has considered models of evolutionary change that track how the semantic distinctions in kin terms change separately from each other. By using statistical inference techniques to reconstruct the evolution of the distinctions from the ground up, we can then later assess whether they have independent trajectories of change, or if they are linked by dependencies-bound coevolutionarily to one another, and/or to other aspects of language and culture.

In terms of ancestral states, sibling terms for early Austronesian speech communities have been well-reconstructed by the (linguistic) comparative method for some time. Milke (1938) reconstructed four terms for Proto Oceanic: *tansi, "younger same-sex sibling," *kaka "older same-sex sibling," *mwane "woman's brother," and *papine, "man's sister." Blust (1980, 1994) reconstructed four terms for Proto Malayo-Polynesian: *ñaRa, "woman's brother," *betaw "man's sister," *kaka "older sibling," and *hua(n)ji, "younger sibling," with the last two possibly restricted to same-sex siblings only. The inference then is that PMP made a relative age and a relative sex distinction based on the sex of the speaker, and that this was carried through to POC. Fox (1994) notes however that different typologies or meaning sets can be extracted from the same 
sets of lexical forms reconstructed for $\mathrm{PMP}^{1}$; furthermore, historical linguistics has no accepted statistical basis for assigning meaning to reconstructed forms. Thus, phylogenetic techniques can be used to effect when the historical linguistic approach may not be sufficient to robustly reconstruct the semantics underlying kin classification.

The Present Study. Here I show how phylogenetic comparative methods can reconstruct ancestral states of sibling patterns in kin terminology. The units to be reconstructed are the distinctions in meaning: the presence or absence of (1) relative age and (2) relative sex. I infer the probability of these distinctions at three main nodes of interest representing important ancestral speech communities (PAN, PMP, POC) and a further six other ancestral nodes. Evolutionary models of transition (gains and losses) in these distinctions are derived. To test the idea of sequential models of change in kin terms more specifically I examine the elaboration and reduction of the opposite-sex distinction. Epling et al. and Marshall both assert that single-step changes are most likely; the idea is implicit in other "lattice" models (e.g., Hage 2001). The prediction is that an opposite-sex distinction (i.e., having one term for opposite-sex siblings and another for same-sex siblings) could evolve from a situation where the distinction was absent. Further, the opposite-sex term could then be elaborated by the sex of speaker, i.e., it could be split into a term for "woman's brother" and "man's sister." However, how often languages then collapse this elaboration back into the single term, or skip straight from absence of the distinction to a two-term system, is unknown. Multistate evolutionary models can quantitatively address the likelihood of these transitions on a phylogeny.

\section{Methods}

Phylogenetic Trees. A posterior distribution (i.e., a probability sample) of 1,000 language trees was derived from a phylogenetic analysis of the Austronesian Basic Vocabulary Database (ABVD) described in Greenhill et al. (2008). The ABVD consists of the cognate sets of a 210-item word-list from more than 600 Austronesian languages. Cognate words are those which can be shown to share a common ancestor by virtue of both similar form and meaning, and are judged as such using the Comparative Method of historical linguistics (different from phylogenetic comparative methods). Each item of basic vocabulary was represented by a number of cognate sets, and set membership was scored for presence or absence for each language, then coded into a binary matrix for the phylogenetic analysis. The kin terms analyzed below are not part of the basic vocabulary used in building the lexical trees themselves.

${ }^{1}$ Fox (1994, p. 138-9) describes two different configurations of meaning for the reconstructed PMP kin terminology vocabulary. The relevant sibling terms are *kaka/aka and *huaji; in the first configuration they can refer to older and younger sibling, respectively, in the second configuration, to older same-sex and younger same-sex, with two extra forms for "brother" and "sister." 


\section{a relative age}

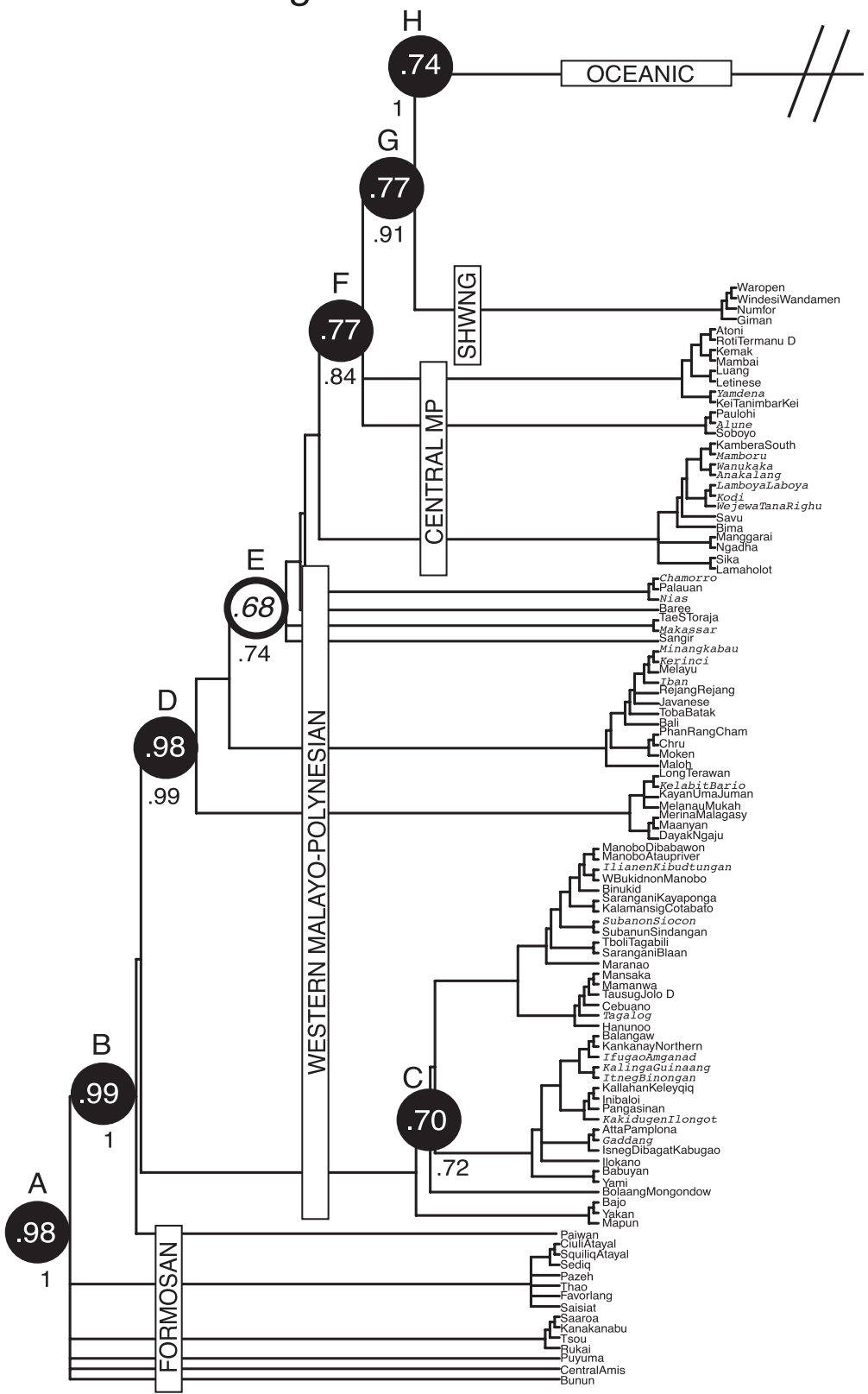

Figure 2. Ancestral state reconstructions of the relative age (a) and relative sex (b) distinction in Austronesian sibling terms. The 50\% majority rule consensus phylogeny summarizes the sample of 1,000 trees for 208 Austronesian languages. Language font indicates the presence (sans-serif) or absence (italic) of the age/sex distinction. Reconstructed nodes are shown by circles coloured (presence $=$ black, absence $=$ grey) according to the dominant ancestral 


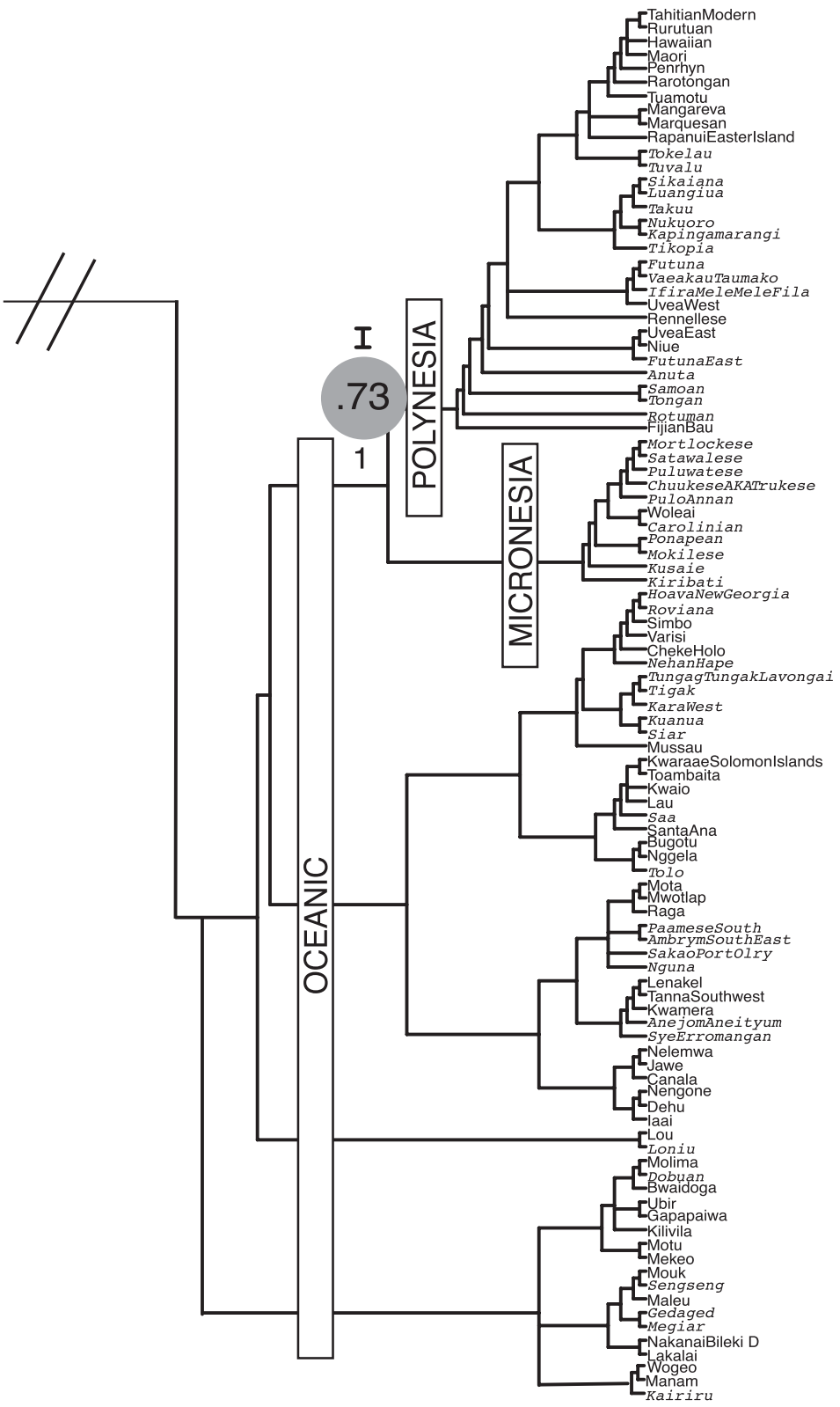

Figure 2. (continued) state reconstruction with the combined posterior probability inside, and lettered to correspond to the distributions in Figures 3 and 4. Filled circles indicate combined probabilities $>.7$, unfilled indicate combined probabilities between .5 and .7. Values below each node indicate the node's phylogenetic posterior probability. Linguistic subgroupings are indicated by white bars crossing branches. Abbreviations: MP, Malayo-Polynesian; SHWNG, South Halmahera-West New Guinea. 


\section{b relative sex}

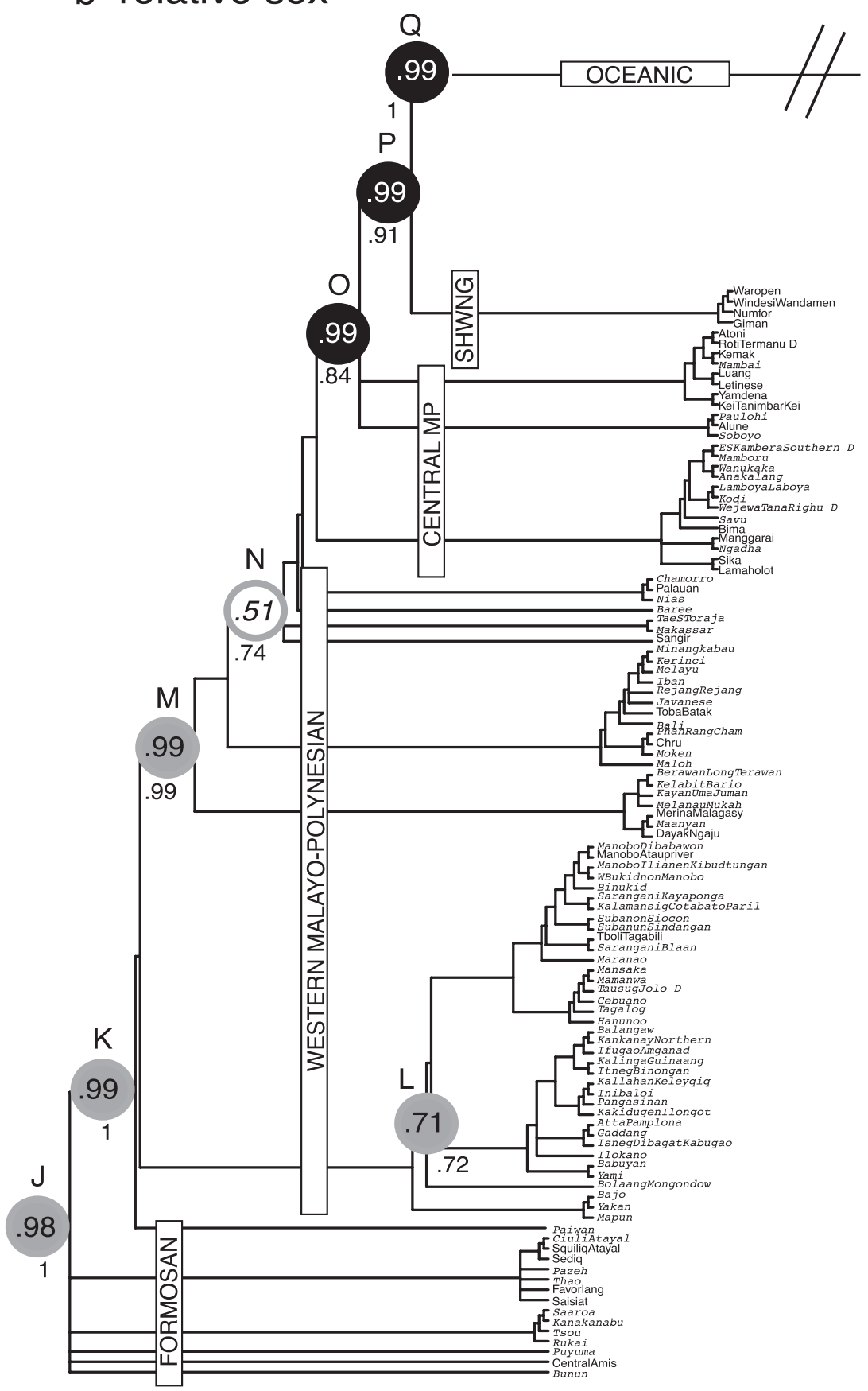

Figure 2. (continued) 


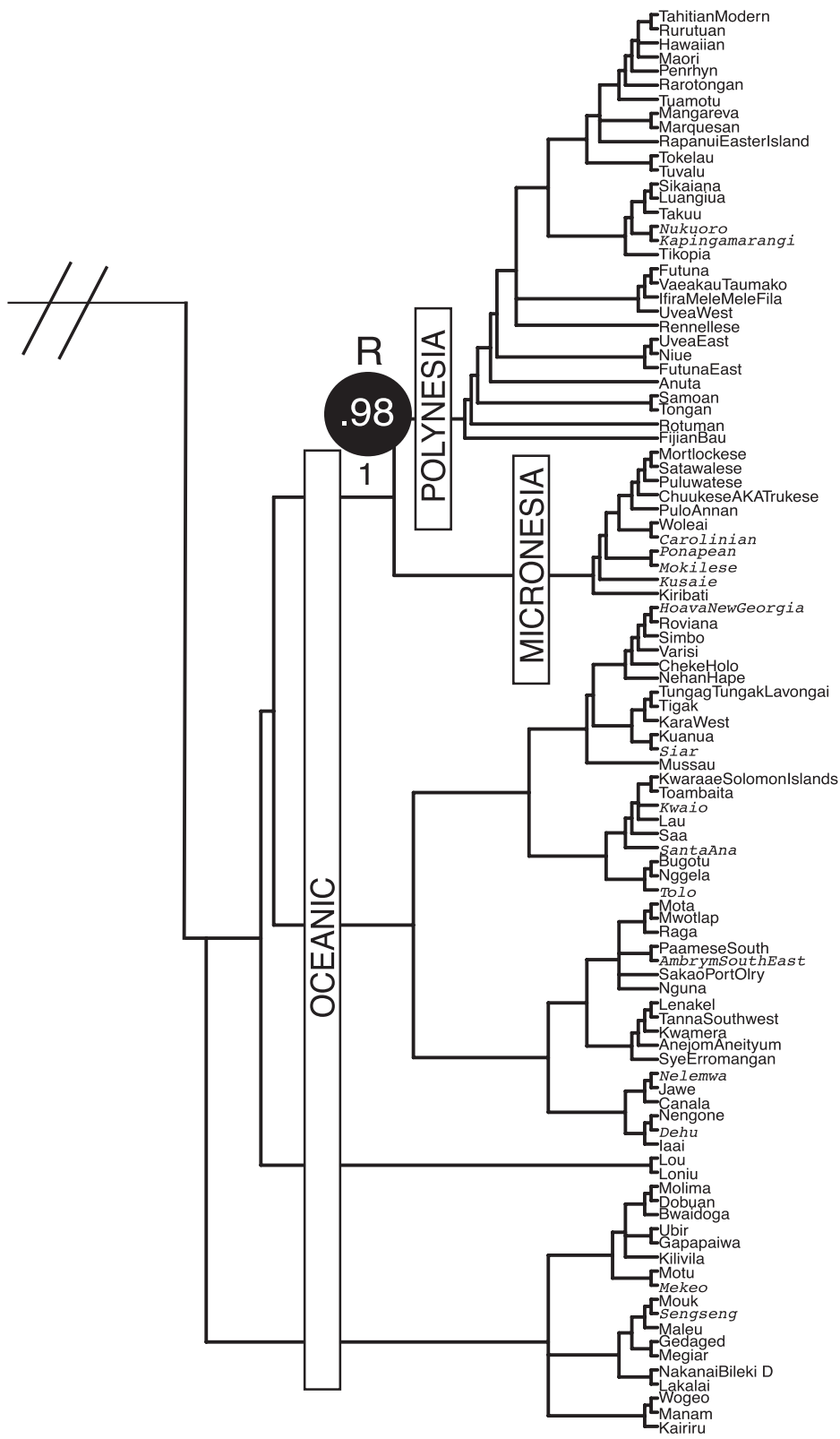

Figure 2. (continued)

The posterior distribution of trees was inferred from these data using Bayesian Markov-chain Monte Carlo (MCMC) methods (Huelsenbeck et al. 2001; Yang and Rannala 1997). This approach makes it possible to account for 
Table 2. Coding Schema for Comparative Analyses

\begin{tabular}{ll} 
Aspect & Description \\
\hline Relative sex & 1 = Language contains terms that distinguish same-sex from \\
& opposite-sex siblings \\
0 & $=$ No same-opposite distinction \\
1 & $=$ Language contains terms that distinguish older and younger \\
& siblings \\
0 & $=$ No older-younger distinction \\
Relative age & sanguage has separate terms denoting the opposite-sex \\
& sibling for each sex (e.g. Woman's brother, man's sister) \\
1 & $=$ Language has a single term for opposite-sex sibling, used \\
Elaboration of cross-siblings ${ }^{\mathrm{a}}$ & by both sexes \\
0 & $=$ No same-opposite distinction
\end{tabular}

a. $n=180$. Languages with intermediate distinctions (e.g., term for "woman's brother" only) were not used in this analysis; a model with five states failed to converge on consistent likelihood estimates.

the effect of uncertainty in the phylogenetic tree model representing population history, a nontrivial consideration in the study of cultural traits because a single branching tree is unlikely to accurately represent human population history (Boyd et al. 1997). The languages $(n=400)$, the trees, and their properties are described more fully in Gray et al. (2009). Retaining those taxa for which corresponding kin term data exists $(n=208)$, a sample of 1,000 trees was used for the comparative analyses below. In this sample, properties of the trees such as branching patterns and branch lengths were represented in proportion to their likelihood. A majority-rules consensus tree was computed in APE Version 2.6 (Paradis et al. 2004) and summarizes the sample visually (see Figure 2), but the comparative analyses were performed over all 1,000 trees.

Kin Terminologies. Data were taken from Dziebel's (2009) worldwide sheets of kin terminologies available at http://kinshipstudies.org/?page_id=20. I matched languages from the ABVD to languages in this data set on the basis of language name and reference source, obtaining a final sample of 208 languages on the phylogenies with sibling terminology data. Languages were coded under the schemes described in Table 2. Terms were coded without in-depth linguistic analysis, but here the investigation is confined to the evolution of age and sex distinctions expressed in the sibling terms themselves, rather than in the language at large, so forms which are made up of morphemes with age- or sex-related meanings are treated the same as other forms. I note here that the analyses concentrate on the distinctions in meaning: the investigation of coevolution in forms and meanings (the traditional purvey of historical linguistics) is a future study. In Table 2, the "elaboration of opposite-sex distinctions" scheme was included as a way to test a general hypothesis about kin-term evolution.

The maps in Figure 1 show the distribution and location of the 208 languages, coded for (1) the relative age distinction and (2) relative sex distinction. 
Comparative Analyses. To analyze the evolution of the sibling term distinctions I used the phylogenetic comparative method Multistate implemented in the BayesTraits package from http://www.evolution.rdg.ac.uk/BayesTraits (Pagel 1999b; Pagel and Meade 2006; Pagel et al. 2004). This package can be used to conduct both maximum-likelihood (ML) and Bayesian analyses. ML analyses were performed initially to explore the data and to assess the range of the model parameters for further Bayesian analyses.

Bayesian approaches to phylogenetic analysis are increasingly preferred in evolutionary biology because, given the data and a set of phylogenetic trees, the methods allow for the estimation of posterior probability distributions of parameters of interest. In other words, they estimate distributions of the ancestral state probabilities at internal nodes, and the rates of change in traits, rather than single values of these parameters. The posterior probability of a parameter value is a quantity proportional to its likelihood of having produced the observed data and represents the probability of the parameter value given the data and the model of trait evolution (Lewis 2001; Ronquist 2004). Because posterior probabilities cannot feasibly be computed analytically, posterior probability distributions are inferred using an MCMC sampling algorithm. This distributional approach provides information about the degree of statistical uncertainty in the cultural trait reconstructions. Crucially, the estimation of parameters over a probability sample of trees yields estimates that are not dependent on any specific phylogenetic hypothesis. Finally, parameters can be estimated over different models of trait evolution, and this then yields estimates that are not dependent on any specific model of how the traits have evolved.

Given the comparative data and tree sample, BayesMultistate uses a continuous-time Markov model to describe the evolution of the trait of interest along the branches of a phylogeny. Under this model, the trait describing (for example) a relative age distinction in sibling terms can switch repeatedly between its two states of $0=$ absence and $1=$ presence in any of the branches of a tree. Two rate parameters, with rate mathematically denoted as $q$, specify the rate of change from one state to another: the transition between 0 and 1 is denoted $q_{01}$, and that between 1 and 0 as $q_{10}$. These rates define the probabilities of each of these changes and therefore both the character states at internal nodes on a tree and the overall likelihood of the data (Pagel 1994, 1999b). The Bayesian MCMC implementation of Multistate estimates the posterior probability distributions of rate parameters and ancestral character states (Pagel and Meade 2006; Pagel et al. 2004). The MCMC chain explores parameter space widely, randomly modifying these parameters and trying them out on the sample of trees over consecutive iterations. While the chain attempts to maximize the likelihood, it also accepts less-likely combinations of parameters at a frequency proportional to their likelihood. After an initial period of "burn-in," the chain reaches stationarity, wandering around a mean likelihood value and accepting the parameter hypotheses into the posterior distribution. This distribution then contains combinations of parameters such that those with higher support are sampled to a greater extent. 
"Reversible-jump" mode was used to additionally estimate the posterior probability distribution of all the possible models of trait evolution specified by the two rate parameters (Pagel and Meade 2006). Let us suppose that we obtain a value of 5 for $q_{01}$ and 7 for $q_{10}$. By then setting these two rates to be equal to each other, and re-running our analysis, we can test whether they are indeed equivalent by comparing the new likelihood score to the old. The reversible-jump procedure outputs a model string describing these equivalences in the rate parameters for us, such that rates are assigned to equivalence classes (denoted by ordered integers) or a "zero bin" depicted by Z. For example, the model string "01" assigns $q_{01}$ to a rate class (0) and $q_{10}$ to a class (1): these two rates are therefore "significantly" different. Another model string "Z0" indicates that rate $q_{01}$ is set to zero while $q_{10}$ takes a non-zero value. For analyses with more than two states, the reversible-jump procedure can reduce the complexities of the underlying model of evolution to tractable dimensions, so it was used here in the three-state analysis of the elaboration of opposite-sex sibling terms.

For each of the sibling term coding schemes three MCMC chains were run for $10^{9}$ iterations. Transition-rate parameters and ancestral state estimates were recorded from the chain into the posterior distribution every 10,000 generations to reduce autocorrelation between iterations. Burn-in was determined empirically by examining a plot of the likelihood against the iteration in Tracer (Rambaut and Drummond 2007), and a posterior of $5 \times 10^{5}$ samples was taken from the stationary part of the chain. The harmonic mean of the likelihoods in the MCMC chain was used to estimate the marginal likelihood of the posterior (Nylander et al. 2004), and the chain with the median marginal likelihood was used for further analyses. In Bayesian phylogenetic analysis one can specify "priors," that is, knowledge that is independent of the data. Often the investigator has no knowledge about, for example, what values are likely for the parameters in the evolutionary model, so a solution is to draw these values from a wide distribution. I compared the marginal likelihood of chains using both uniform "flat" and exponential distributions for priors; both were equivalent and produced posteriors that contained the ML solutions, but chains using an exponential prior (with a mean taken from a distribution on the interval $0-10$ ) reached convergence more quickly and were used for that reason alone.

To estimate ancestral states, the means of the posterior probability distribution of ancestral states at specified internal nodes tree are combined with the posterior probability of each node on the phylogeny; this is denoted as $p$ (node) and represents the probability that the node exists. For example, for a specific node BayesMultistate may return a posterior probability distribution with a mean of 0.8 for the presence of a relative age distinction; this is denoted $p$ (Age I node). If the node is present in all trees (i.e., $p$ [node] $=1.00$ ), we accept the 0.8 value as the posterior probability of the age distinction at that node. However, if the node is only present in $60 \%$ of the trees (i.e., $p[$ node] $=0.60$ ), we report the "combined probability" for the relative age distinction, $p($ Age $)=p($ Age $\mid$ node $) * p($ node $)=0.8 * 0.6=0.48$. A value of 0.7 for the combined probabilities represents an acceptable value of 

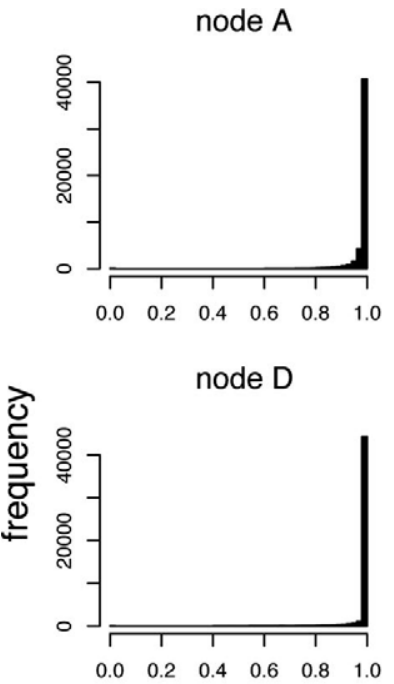

node $\mathrm{G}$

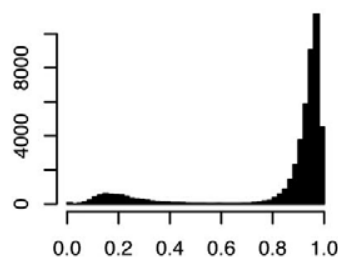

node B

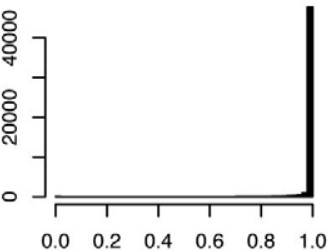

node $\mathrm{E}$

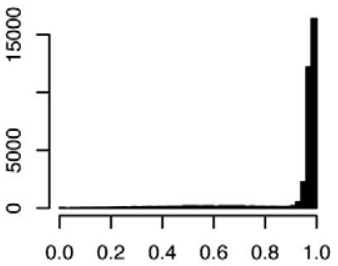

node $\mathrm{H}$
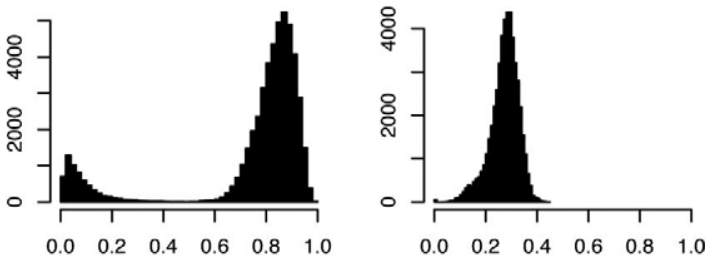

\section{probability (Age)}

Figure 3. Posterior probability distributions of reconstructed ancestral states for the presence of the relative age distinction. Each distribution is lettered according to the corresponding major node in Figure 2a. Abbreviations as for Figure 2.

certainty for an ancestral state at a node (M. Pagel, personal communication). Node values are reported as means $\pm \mathrm{SD}$, and marginal likelihoods are reported with the 95\% highest probability density (HPD) range (i.e., the range within which $95 \%$ of the posterior distribution falls).

\section{Results}

Ancestral States. Ancestral state reconstructions (means) derived from the Bayesian MCMC analysis of relative age and relative sex distinctions are shown at the appropriate nodes on the phylogenies in Figure 2. The histograms in Figure 3 (relative age) and Figure 4 (relative sex) show the probability distributions at each node for the presence and absence of the two distinctions, demonstrating the 
node $\mathrm{J}$

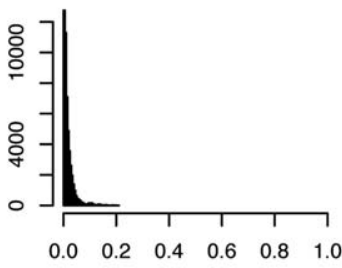

node $\mathrm{M}$

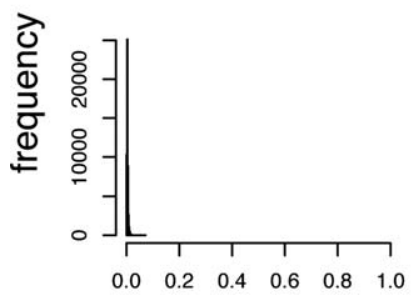

node $\mathrm{P}$

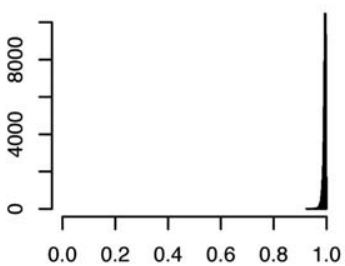

node $\mathrm{K}$
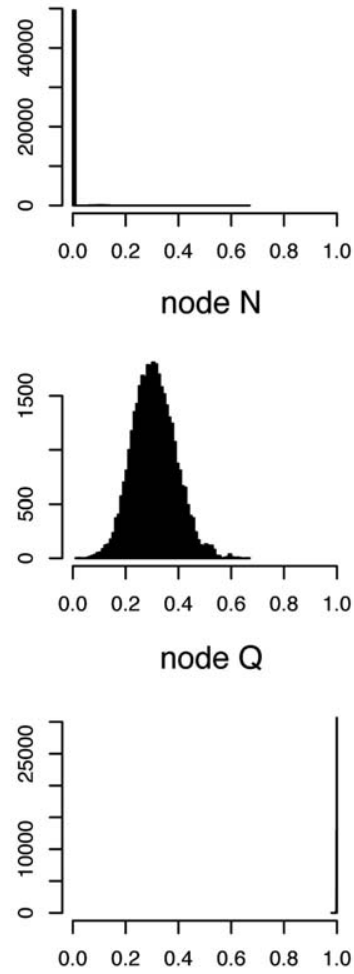

probability (Sex)

node L
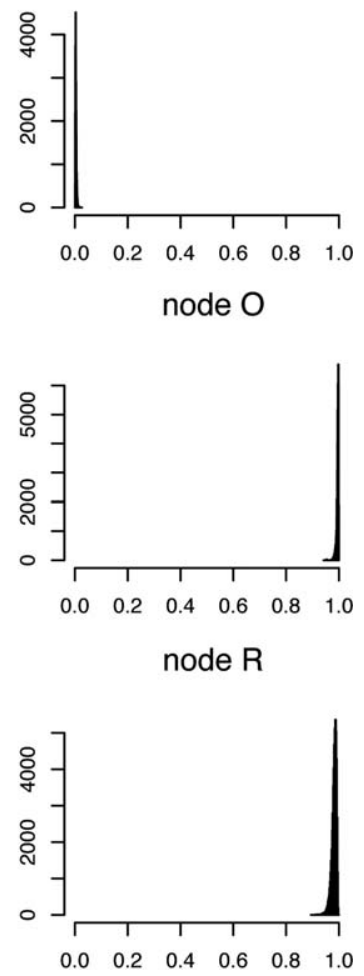

Figure 4. Posterior probability distributions of reconstructed ancestral states for the presence of the relative sex distinction. Each distribution is lettered according to the corresponding major node in Figure 2b. Abbreviations as for Figure 2.

variable degree of robustness in the mean estimates shown in Figure 2. The three figures should be read in conjunction.

Relative Age. The mean marginal likelihood was -116.01 (95\% HPD = -118.5 to -111.6$)$. The relative age distinction is securely reconstructed as present for the root, $\mathrm{PAN}(p($ Age $\mid$ node $\mathrm{A})=p($ Age $)=0.98 \pm 0.02)$, and throughout most of the high-order nodes reconstructed here. Phylogenetic uncertainty in node $\mathrm{E}$ means that the age distinction cannot be reliably reconstructed here, though the reconstruction itself has $p($ Age $\mid$ node $\mathrm{E})=.92 \pm .07$ in the trees that have the node. The pattern here implies that languages without the relative-age distinction are likely to be later recurrent losses (such as in Micronesian, and some Central Malayo-Polynesian languages). An exception is Proto Central Pacific (node I: Fiji, Rotuma, and Polynesian), where $p($ Age $\mid$ node $\mathrm{I})=p($ Age $)=0.27 \pm 0.26$. 
Relative Sex. The mean marginal likelihood was -96.5 ( -96.8 to -96.6 95\% HPD). The relative sex distinction is not reconstructed for PAN $(p($ Sex $\mid$ node $\mathrm{A})=p($ Age $)=0.02 \pm 0.02), \mathrm{PMP}$, node $\mathrm{L}$ (ancestral to a large group of Greater Central Philippines languages), or node M. The relative sex distinction begins to appear reliably between nodes $\mathrm{M}$ and $\mathrm{N}$, though both phylogenetic and character uncertainty in node $\mathrm{N}$ makes the exact dynamic unclear, $p(\operatorname{Sex} \mid$ node $\mathrm{N})=.69 * .74=.51$. It is then present in all other estimated nodes, becoming unambiguously present through the family into the Oceanic languages e.g., POC $p(\operatorname{Sex} \mid$ node $\mathrm{Q})=p(\operatorname{Sex})=0.99 \pm 0.00$.

\section{Models of Change}

Relative Age. ML estimates of the transition rate parameters over the 1,000 trees ranged between 1.00 and 1.19 for $q_{01}$ (the gain of the age distinction) and .37 and .44 for $q_{10}$ (the loss of the age distinction) with no overlap, indicating a model where the gain of the distinction was more than twice as frequent as its loss. However, the Bayesian analysis found that rates of gain and loss in the relative age distinction were virtually identical: $q_{01}=.30(.14-.6395 \%$ HPD) and $q_{10}=.25$ (.14 - .37 95\% HPD). Bayesian inferences are independent from any one particular phylogenetic hypothesis, so it would appear that the different gain/loss estimates under ML are attributable to differences in tree topologies and (most likely) branch lengths. The reversible-jump procedure, which integrates out over the models themselves (that is, it reduces the "numbers" in the transition rates to qualitative "classes"), also supported a model of equal gains and losses: $q_{01}=q_{10}$, and these models accounted for $87 \%$ of all models visited in the chain. Models where gains were greater than losses (i.e., $q_{01}>q_{10}$ ) comprised the vast majority of other models, and had a higher overall likelihood ( -112.8 compared to -116.5 ), but as mentioned, were apparently dependent on the particular phylogenetic hypothesis.

Relative Sex. ML estimates of the transition rate parameters over the 1,000 trees ranged between .35 and .46 for $q_{01}$ (gain of the sex distinction) and .30 and .35 for $q_{10}$ (loss of the sex distinction), with no overlap, indicating that the gain of the distinction was (just) more frequent than loss. However, the Bayesian analysis again found that rates of gain and loss in the relative sex distinction were virtually identical: $q_{01}=.18(.12-.2695 \%$ HPD $)$ and $q_{10}=.19(.12-.2695 \%$ HPD $)$. Here the reversible-jump procedure supported the model of equal gains and losses: $q_{01}=q_{10}$, and these models accounted for over $99 \%$ of all models visited in the chain. We can thus be certain, independent of the tree topology, branch lengths, and absolute rates of change, that equal gains and losses are appropriate for these data.

Elaboration of Opposite-Sex Sibling Terms. The flow diagrams in Figure 5 show a summary of (1) the maximum-likelihood solutions and (2) the mostvisited models found by reversible-jump Bayesian MCMC. The ML summary is 

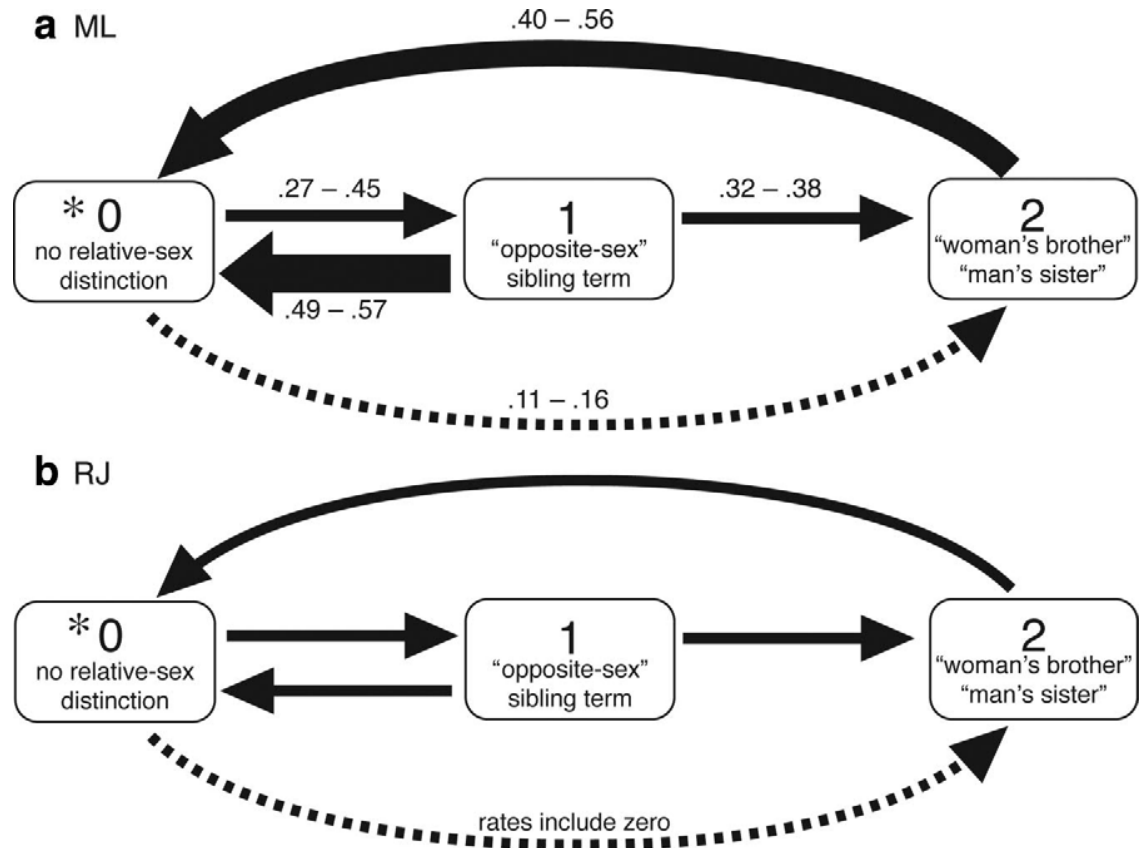

Figure 5. Models of evolution describing the elaboration of the opposite-sex sibling term. (a) The ranges of the ML solutions for each transition, found over 1,000 trees. (b) The most-visited model using RJ MCMC. The asterisked state indicates the root reconstruction. See text for details.

annotated with the ranges of the transition-rate parameters for each possible change. The Bayesian summary depicts the top three models, which together comprise more than $70 \%$ of all models visited by the RJ MCMC chain. The first accounted for 33\% of the MCMC chains (i.e., it was the most-visited model): this model has equal rates for all transitions except for $q_{21}$, (a change from two terms "woman's brother"/“man's sister" back to a single term "opposite-sex sibling") which is set to zero. The second most-visited model, accounting for $25 \%$ of the total, proposed equal rates for all transitions, while the third (13\%) assigned $q_{02}$ (from no distinction, to the two-term system) to the zero bin and set all others to equal.

These analyses suggest that the most likely evolutionary pathway was one where Austronesian languages started with no relative-sex distinction in PAN (as above), gained the single term "opposite-sex sibling," and then this was elaborated into two terms "woman's brother"/"man's sister" in a number of linguistic subgroups. Jumps from the absence of the distinction to the two-term situation $\left(q_{02}\right)$ were rare and often zero; the collapse of "woman's brother"/"man's sister" back to the single "opposite-sex sibling" term $\left(q_{21}\right)$ were all zero under ML, and rare in the Bayesian analysis. Both analyses also show high rates of loss of any form of the opposite-sex distinction overall 
(i.e., $q_{20}$ and $q_{10}$ ). When all models are considered, those where $q_{21}$ is in the zero bin have a higher marginal likelihood than those where it has a non-zero rate, providing support for the hypothesis that collapses of an elaborated term are rare.

\section{Discussion}

Using phylogenetic comparative methods, I inferred the ancestral states of two important distinctions found in Austronesian sibling terms: relative age and relative sex. The age distinction was reconstructed for the earliest nodes of the tree, indicating that languages without the distinction lost it later, while the sex distinction could only be reconstructed firmly to the ancestor of Eastern Malayo-Polynesian languages: here, PAN, PMP and some early Philippine (node L) speech communities did not have this distinction. Both distinctions could be adequately described by a model of change in which gains and losses were equally likely.

In the past, the study of kin term semantics has either been within a typological framework, which carries an inherent amount of arbitrariness in the creation of type-sets (e.g., "Iroquois cousin terminologies") and/or approached with the tools of componential analysis, which uses distinctions like those used here in the service of describing meaning by formal logic, without any diachronic considerations of the evolutionary processes of change. The models of change used here trace the independent histories of the age- and sex-distinctions, and do not rely on typologies. Further, they are independent of any particular phylogenetic hypothesis about prehistory. Because they are integrated over the range of statistically plausible linguistic relationships that the data supports, they thus incorporate uncertainty in a similar fashion to network models of ethnolinguistic relatedness. Phylogenetic (tree) and character (sibling term distinction) uncertainty can be separated, so for the subset of trees that do have a particular node (such as node E/N) relative age can be securely reconstructed whereas the presence of relative sex is ambiguous: these nodes can then be targets for further in-depth lexical analysis.

The reconstructions for the relative age distinction agree with the lexicalsemantic reconstructions from historical linguistics that posit relative age (younger sibling/older sibling) for PMP and POC (Blust 1994), and those from comparative ethnography that infer the same for ancestral Philippines (Kroeber 1919). However, the phylogenetic reconstructions of relative sex do not agree with historical linguistic reconstructions for PMP: they are robustly absent in the former but present by the latter method. A possible explanation for this may be the large number of Formosan and Western Malayo-Polynesian languages that lack the relative sex distinction in meaning but (in the WMP case) contain occasional reflexes of a PMP sibling term. Arbitrating this conflict, and the similar case for Proto Central Pacific relative age (see Figure 2a, node I), will require a word-meaning coevolutionary approach, where we test how the reconstructed distinctions are coupled to the evolutionary trajectories of the 
lexical forms. Thus, future work will require historical linguistic analysis to judge the cognacy of the lexical forms, using the linguistic comparative method (LCM). This is crucial: those authors who took a solely structural approach to Oceanic sibling term systems (Epling et al. 1973; Marshall 1984) were criticized for their dismissal of the lexical evidence and the logic of the LCM (see the comments to Marshall, 1984 by Blust, Clark, and Chowning).

One tenet of the LCM is that convergence (independent innovation) of the same forms with the same meanings in different languages is vastly improbable: explanations based on inheritance or borrowing (i.e., transmission) are preferable in such cases. Phylogenetic coevolutionary analyses can test how lexical forms are yoked together with semantic distinctions: we will be able to identify when independent gains of a distinction in different languages should be invoked as explanation, or when transmission with frequent loss is preferred. We shall also be able to test meaning-meaning coevolution, that is, whether semantic changes become so tightly coupled they happen in lockstep. For example, it may be that a change in lexical form for a sibling term packages together two or more aspects of meaning, so (e.g.) age and sex distinctions become transmitted as a single unit. However, the merit of the initial approach taken here is that by treating the distinctions as the unit of analyses, against the background of the linguistic phylogeny, we are afforded insight into the loss and gain of the meanings themselves independent of the lexical items used.

The model of change underlying the elaboration of the opposite-sex distinction suggests that some transitions in kin terms are not possible. Jumps from no distinction to two terms for opposite sex siblings based on sex of speaker are very unlikely. Once gained, the two terms are also unlikely to collapse back into the single term for opposite-sex sibling. The evolutionary schematic of Epling et al. (1973) proposed that any sibling terminology type could be derived iteratively from another through "a process of making new binary distinctions and/or removing old ones" (p. 1602); the model here supports the first assertion but not the second. Blust (1994) suggested that the loss of distinctions will occur more frequently than their gain, especially if correlated with cognate forms, but the model in Figure 5 supports the idea that while losses of a distinction altogether are likely, piece-wise dismantling are not. Repeating these analyses and the extensions described above in other language families for which phylogenies are available (Indo-European and Bantu) will reveal whether the underlying models of change are lineage-specific or more general, cf work on post-marital residence patterns comparing Austronesian and Indo-European (Fortunato and Jordan 2010).

Perspectives. One of the oldest debates in anthropology concerns the extent to which changes in kin terms reflect changes in the social structure of the language community-Rivers (1914) "functional" view-versus Kroeber's (1909) statement that kin terms reflected only psychological and linguistic systems and were not correlated with ecological or social-structural pressures. Revisiting kin term systems armed with the investigative tools of evolutionary biology means that we 
may make some headway on this debate. If as Rivers asserted, and Murdock (1949, 1968) later tabulated considerable cross-cultural data in support of, there are consistent relationships between social structure and kin term systems, we shall now be able to test these hypotheses with adequate control for Galton's Problem. Many implicational universals in anthropology, for instance that of "Type F" relative-age sibling terms and the presence of descent groups (Murdock 1968), rest on simple association data that do not control for evolutionary history. Extrapolation from these associations back into the past in support of claims that pre-historical communities had some or other form of social structure (Blust 1980; Ehret 2008) can thus shape ideas (possibly erroneously) about past population movements and the interpretation of human genetic biogeography (Wilkins and Marlowe 2006).

Unlike earlier work, no inference about population history is or should be made from the kin terms themselves. Multiple distinct transmission pathways may characterize the members of any set of cultural and/or linguistic features (Boyd et al. 1997), and methods exist to quantify the degree to which those histories overlap, usually by partitioning data (Matthews et al.) or examining the congruence between trees produced from different data set (Page 2003; Tehrani et al. 2010). However, because kin terms constitute internally correlated systems, building phylogenies from these data is problematic. Instead, by tracking the semantic distinctions on core vocabulary phylogenies, we may be confident that similar dynamics of linguistic transmission encompass both "trait" and "tree." Furthermore, we can quantify the phylogenetic signal (Ives and Garland 2009; Pagel 1999a) in aspects of social structure (e.g., descent groups, marriage patterns) to ascertain the overlap in transmission pathways between culture and language (Mace and Jordan 2011).

Finally, an understanding of the evolution of kin terminologies carries implications beyond anthropology for cognitive science (Jones 2010). For example, the distinctions commonly found in sibling terminologies have psychological import: linguistic semantic categories such as age and sex can influence nonlinguistic processing in judgment, production, and memory (Anggoro and Gentner 2003), thus potentially speaking to the debate in the cognitive sciences as to the degree to which language and thought are related (Gumperz and Levinson 1991). Witkowski (1972) remarked that the size, complexity, and coverage of the $1,000+$ kin terminologies known to science were a "precious resource" for the strategic study of semantic change. Combined with the Darwinian realities of relatedness, group membership, and reciprocity that they filter, kin terms provide a unique focus for understanding the evolution of cultural and linguistic diversity (Jordan and Dunn 2010).

Acknowledgments I thank the members of EPLC and the Language and Cognition Group at the Max Planck Institute for Psycholinguistics, as well as three anonymous referees, for their useful comments; Russell Gray, Simon Greenhill, and Robert Blust for 
making the Austronesian phylogenies available; and German Dziebel for providing the kin term data. This research was supported by the Max Planck Society.

Received 1 April 2010; revision accepted for publication 28 July 2010.

\section{Literature Cited}

Anggoro, F., and D. Gentner. 2003. Sex and seniority: The effects of linguistic categories on conceptual judgments and memory. In Proceedings of the Twenty-Fifth Annual Conference of the Cognitive Science Society. R. Alterman and D. Kirsh, eds. Boston, MA: Lawrence Erlbaum.

Ben Hamed, M. 2005. Neighbour-nets portray the Chinese dialect continuum and the linguistic legacy of China's demic history. Proc. Royal Soc. B Biol. Sci. 272:1015-1022.

Blust, R. 1980. Early Austronesian social organization: The evidence of language. Curr. Anthropol. 21:205-247.

Blust, R. 1994. Austronesian sibling terms and culture history. In Austronesian Terminologies: Continuity and Change, Pacific Linguistics, A. Pawley and M. D. Ross, eds. Canberra, Australia: Australian National University Press; 31-72.

Boyd, R., M. Borgerhoff-Mulder, W. H. Durham et al. 1997. Are cultural phylogenies possible? In Human By Nature: Between Biology and the Social Sciences. Hillsdale, NJ: Lawrence Erlbaum. 355-386.

Collard, M., S. J. Shennan, and J. J. Tehrani. 2006. Branching, blending, and the evolution of cultural similarities and differences among human populations. Evol. Hum. Behav. 27:169-184.

Colleran, H., and R. Mace. 2011. Contrasts and conflicts in anthropology and archaeology: The evolutionary/interpretive dichotomy in human behavioural research. In Darwinian Archaeologies: A Discussion, E. Cochrane and A. Gardner, eds. Walnut Creek, CA: Leftcoast Press.

D'Andrade, R. 2000. The sad story of anthropology 1950-1999. Cross-Cultural Research 34:219-232.

Diamond, J., and P. Bellwood. 2003. Farmers and their languages: The first expansions. Science 300:597-603.

Dunbar, R. I. M., and L. Barrett, eds. 2007. Oxford Handbook of Evolutionary Psychology. Oxford, U.K.: Oxford University Press.

Dziebel, G. V. 2009. Kinship studies. http://kinshipstudies.org. Accessed 07/04/2009.

Ehret, C. 2008. Reconstructing ancient kinship in Africa. In Early Human Kinship: From Sex to Social Reproduction, N. J. Allen, H. Callan, R. I. Dunbar, and W. James, eds. Oxford, U.K.: Blackwell; 200-231.

Epling, P., J. Kirk, and J. P. Boyd. 1973. Genetic relations of Polynesian sibling terminologies. Am. Anthropol. 75:1596-1625.

Firth, R. 1936. We, the Tikopia. London, U.K.: Allen and Unwin.

Fortunato, L., and F. M. Jordan. 2010. Your place or mine? A phylogenetic comparative analysis of postmarital residence in Indo-European and Austronesian societies. Phil. Trans. R. Soc. Lond. B. 365:3913-3922.

Fortunato, L., and R. Mace. 2009. Testing functional hypotheses about cross-cultural variation: A maximum-likelihood comparative analysis of Indo-European marriage practices. In Pattern and Process in Cultural Evolution, S. J. Shennan, ed. Berkely, CA: University of California Press; 235-249.

Fox, J. J. 1994. Who's who in Ego's generation: Probing the semantics of Malayo-Polynesian kinship classification. In Austronesian Terminologies: Continuity and change, Pacific linguistics, A. Pawley and M. D. Ross, eds. Canberra, Australia: Australian National University Press; $127-140$. 
Fox, J. J., P. Bellwood, J. J. Fox et al. 1995. Austronesian societies and their transformations. In The Austronesians: Historical and Comparative Perspectives. Canberra, Australia: Australian National University Press.

Fox, R. 1967. Kinship and Marriage. Harmondsworth, Middlesex, U.K.: Penguin.

Gray, R. D., and Q. D. Atkinson. 2003. Language-tree divergence times support the Anatolian theory of Indo-European origin. Nature. 426:435-439.

Gray, R. D., A. J. Drummond, and S. J. Greenhill. 2009. Language phylogenies reveal expansion pulses and pauses in Pacific settlement. Science 323:479-483.

Gray, R. D., S. J. Greenhill, and R. M. Ross. 2007. The pleasures and perils of Darwinizing culture (with phylogenies). Biological Theory 2:360-375.

Green, R. C. 2003. The Lapita horizon and traditions-Signature for one set of oceanic migrations. In Pacific Archaeology: Assessments and Prospects: Proceedings of the International Conference for the 50th Anniversary of the first Lapita Excavation, Koné, Nouméa 2002. C. Sand, ed., 95-120.

Greenhill, S. J., R. Blust, and R. D. Gray. 2008. The Austronesian basic vocabulary database: From bioinformatics to lexomics. Evol. Bioinform. 4:271-283.

Gumperz, J. J., and S. C. Levinson. 1991. Rethinking linguistic relativity. Curr. Anthropol. 32:613-623.

Hage, P. 2001. Marking theory and kinship analysis: Cross-cultural and historical applications. Anthropological Theory 1:197-211.

Harvey, P. H., and M. D. Pagel. 1991. The Comparative Method in Evolutionary Biology. New York, NY: Oxford University Press.

Holden, C. J. 2002. Bantu language trees reflect the spread of farming across sub-Saharan Africa: a maximum-parsimony analysis. Proc. Royal Soc. Lond. B Biol. Sci. 269:793-799.

Holden, C. J., and R. Mace. 2003. Spread of cattle led to the loss of matrilineal descent in Africa: A coevolutionary analysis. Proc. Royal Soc. Lond. B Biol. Sci. 270:2425-2433.

Hrdy, S. B. 2009. Mothers and Others: The Evolutionary Origins of Mutual Understanding. Cambridge, MA: Harvard University Press.

Huelsenbeck, J. P., F. Ronquist, R. Nielsen et al. 2001. Bayesian inference of phylogeny and its impact on evolutionary biology. Science 294:2310-2314.

Ives, A. R., and T. Garland, Jr. 2009. Phylogenetic logistic regression for binary dependent variables. Syst. Biol. syp074.

Jones, D. 2003. The generative psychology of kinship: Part 1. Cognitive universals and evolutionary psychology. Evol. Hum. Behav. 24:303-319.

Jones, D. 2010. Human kinship, from conceptual structure to grammar. Behav. Brain Sci. 33:367-416.

Jordan, F. M., and M. Dunn. 2010. Kin term diversity is the result of multilevel, historical processes. Behav. Brain Sci. 33:388.

Jordan, F. M., R. D. Gray, S. J. Greenhill et al. 2009. Matrilocal residence is ancestral in Austronesian societies. Proc. Royal Soc. Lond. B Biol. Sci. 276:1957-1964.

Jordan, P., and S. Shennan. 2009. Diversity in hunter-gatherer technological traditions: Mapping trajectories of cultural "descent with modification" in northeast California. J. Anthropol. Archaeol. 28:342-365.

Kirch, P. V., and R. C. Green. 2001. Hawaiiki, Ancestral Polynesia: An Essay in Historical Reconstruction. Cambridge, U.K.: Cambridge University Press.

Kitchen, A., C. Ehret, S. Assefa et al. 2009. Bayesian phylogenetic analysis of Semitic languages identifies an Early Bronze Age origin of Semitic in the Near East. Proc. Royal Soc. Lond. B Biol. Sci. 276:2703-2710.

Kroeber, A. L. 1909. Classificatory systems of relationship. The Journal of the Royal Anthropological Institute of Great Britain and Ireland 39:77-84.

Kroeber, A. L. 1919. Peoples of the Philippines. New York, NY: American Museum Press.

Kronenfeld, D. B. 2001. Introduction: The uses of formal analysis re cognitive and social issues. Anthropological Theory. 1:147-172. 
Laland, K. N., and G. N. Brown. 2002. Sense \& Nonsense: Evolutionary Perspectives on Human Behaviour. Oxford, U.K.: Oxford University Press.

Lane, R. B. 1961. A Reconsideration of Malayo-Polynesian social organization. American Anthropologist 63:711-720.

Levi-Strauss, C. 1969. The Elementary Structures of Kinship. Boston, MA: Beacon Press.

Lewis, P. O. 2001. Phylogenetic systematics turns over a new leaf. Trends in Ecology \& Evolution 16:30-37.

Lipo, C. P., M. J. O'Brien, M. Collard, and S. Shennan, eds. 2006. Mapping our Ancestors: Phylogenetic Approaches in Anthropology and Prehistory. New York, NY: Aldine.

Lounsbury, F. G. 1987. The structural analysis of kinship semantics. In Cognitive Anthropology, S. Tyler, ed. Long Grove, IL: Waveland Press. 193-211

Mace, R., and C. J. Holden. 2005. A phylogenetic approach to cultural evolution. Trends Ecol. Evol. 20:116-121.

Mace, R., C. J. Holden, and S. Shennan, eds. 2005. The Evolution of Cultural Diversity: A Phylogenetic Approach. London, U.K.: Left Coast Press \& Berg.

Mace, R., and F. M. Jordan. 2011. Macro-evolutionary studies of cultural diversity: A review of empirical studies of cultural transmission and cultural adaptation. Proc. Royal Soc. Lond. B Biol. Sci. 366:402-411.

Mace, R., and M. Pagel. 1994. The comparative method in anthropology. Curr. Anthropol. 35:549-564.

Marshall, M. 1983. Siblingship in Oceania: Studies in the Meaning of Kin Relations. ASAO monograph. Lanham, MD and London: University Press of America.

Marshall, M. 1984. Structural patterns of sibling classification in Island Oceania: Implications for culture history. Curr. Anthropol. 25:597-637.

Matthews, L., J. J. Tehrani, F. M. Jordan et al. In press. Testing for divergent transmission histories among cultural characters: A study using Bayesian phylogenetic methods and Iranian Tribal textile data. PLoS One.

Milke, W. 1938. Die Bennunnungen der Geschwister in den austronesischen Sprachen Ozeaniens. Zeitschrift für Ethnologie. 70:51-66.

Morgan, L. H. 1871. Systems of Consanguinity and Affinity in the Human Family: Contributions to Knowledge. Washington, DC: Smithsonian Institution.

Murdock, G. P. 1949. Social Structure. New York, NY: MacMillan.

Murdock, G. P. 1968. Patterns of sibling terminology. Ethnology 7:1-24.

Nerlove, S., and S. K. Romney. 1967. Sibling terminology and cross-sex behavior. Am. Anthropol. 69:179-187.

Nylander, J. A. A., F. Ronquist, J. P. Huelsenbeck et al. 2004. Bayesian phylogenetic analysis of combined data. Syst. Biol. 53:47-67.

Page, R. D. M., ed. 2003. Tangled Trees: Phylogeny, Cospeciation, and Coevolution. Chicago, IL: University of Chicago Press.

Pagel, M. 1994. Detecting correlated evolution on phylogenies: A general method for the comparative-analysis of discrete characters. Proc. Royal Soc. Lond. B Biol. Sci. 255:37-45.

Pagel, M. 1999a. Inferring the historical patterns of biological evolution. Nature 401:877-884.

Pagel, M. 1999b. The maximum likelihood approach to reconstructing ancestral character states of discrete characters on phylogenies. Syst. Biol. 48:612-622.

Pagel, M., Q. D. Atkinson, and A. Meade. 2007. Frequency of word-use predicts rates of lexical evolution throughout Indo-European history. Nature 449:717-720.

Pagel, M., and A. Meade. 2006. Bayesian analysis of correlated evolution of discrete characters by reversible-jump Markov chain Monte Carlo. Am. Nat. 167:808-825.

Pagel, M., A. Meade, and D. Barker. 2004. Bayesian estimation of ancestral character states on phylogenies. Syst. Biol. 53:673-684.

Paradis, E., J. Claude, and K. Strimmer. 2004. APE: Analyses of phylogenetics and evolution in R language. Bioinformatics 20:289-290.

Parkin, R. 1997. Kinship: An Introduction to the Basic Concepts. Oxford, U.K.: Blackwell. 


\section{Phylogenetic Analysis of Sibling Terminologies / 321}

Pawley, A. 2002. The Austronesian dispersal: Languages, technologies and people. In Examining the Farming/Language Dispersal Hypothesis, P. Bellwood and C. Renfrew, eds. Cambridge, U.K.: McDonald Institute for Archaeological Research; 251-274.

Radcliffe-Brown, A. R. 1952. Structure and Function in Primitive Society. London, U.K.: Cohen \& West.

Rambaut, A., and A. J. Drummond. 2007. Tracer, v 1.4, available from http://beast.bio.ed.ac.uk/Tracer.

Read, D. W. 2001. Formal analysis of kinship terminologies and its relationship to what constitutes kinship. Anthropological Theory. 1:239-267.

Read, D.W. 2007. Kinship theory: A paradigm shift. Ethnology 46:329-365.

Rexova, K., Y. Bastin, and D. Frynta. 2006. Cladistic analysis of Bantu languages: A new tree based on combined lexical and grammatical data. Naturwissenschaften 93:189-194.

Rivers, W. H. R. 1914. Kinship and Social Organisation. London, U.K.: Constable and Co.

Ronquist, F. 2004. Bayesian inference of character evolution. Trends Ecol. Evol. 19:475-481.

Stone, L. 2000. Kinship and Gender: An Introduction, 2nd edition. Boulder, CO: Westview Press.

Tehrani, J. J., and M. Collard. 2009. On the relationship between inter-individual cultural transmission and population-level cultural diversity: A case study of weaving in Iranian tribal populations. Evol. Hum. Behav. 30:286-300.

Tehrani, J. J., M. Collard, and S. Shennan. 2010. The cophylogeny of populations and cultures: Reconstructing the evolution of Iranian tribal craft traditions using trees and jungles. Proc. Royal Soc. Lond. B Biol. Sci. 365:3865-3874.

Tylor, E. B. 1889. On a method of investigating the development of institutions applied to laws of marriage and descent. J. Royal Anthropol. Inst. 18:245-386.

Van Wouden, F. A. E. 1935 [1968]. Types of Social Structure in Eastern Indonesia. The Hague, Netherlands: Nijhoff.

Wierzbicka, A. 1987. Kinship semantics: Lexical universals as a key to psychological reality. Anthropological Linguistics 29:131-156.

Wilkins, J. F., and F. Marlowe. 2006. Sex-biased migration in humans: What should we expect from genetic data. Bioessays 28:290-300.

Witkowski, S. 1972. Guttman scaling of semantic distinctions. In Kinship Studies in the Morgan Centennial Year, P. Reining, ed. Washington, D.C.: The Anthropological Society of Washington; 167-188.

Yang, Z. H., and B. Rannala. 1997. Bayesian phylogenetic inference using DNA sequences: A Markov chain Monte Carlo method. Mol. Biol. Evol. 14:717-724. 